\title{
Clinical Characteristics and Prognostic Factors of Lung Cancer in Korea: A Pilot Study of Data from the Korean Nationwide Lung Cancer Registry
}

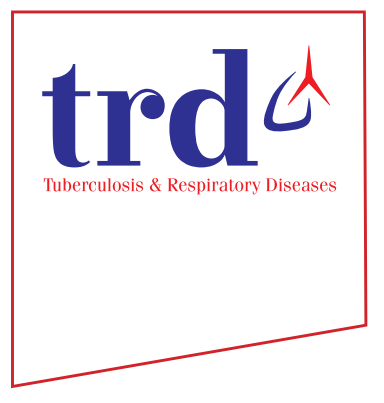

Ho Cheol Kim, M.D. ${ }^{10}$, Chi Young Jung, M.D., Ph.D. ${ }^{2}$, Deog Gon Cho, M.D., Ph.D. ${ }^{3}$, Jae Hyun Jeon, M.D. ${ }^{4}$, Jeong Eun Lee, M.D., Ph.D. ${ }^{5}$, Jin Seok Ahn, M.D., Ph.D. ${ }^{6}$, Seung Joon Kim, M.D., Ph.D. ${ }^{7}$, Yeongdae Kim, M.D., Ph.D. ${ }^{8}$, Young-Chul Kim, M.D., Ph.D. ${ }^{9}$, Jung-Eun Kim, B.S. ${ }^{10}$, Boram Lee, M.S. ${ }^{10}$, Young-Joo Won, Ph.D. ${ }^{10}$ and Chang-Min Choi, M.D., Ph.D. ${ }^{1,11}{ }^{(1 D}$

${ }^{1}$ Department of Pulmonary and Critical Care Medicine, Asan Medical Center, University of Ulsan College of Medicine, Seoul, ${ }^{2}$ Department of Internal Medicine, Catholic University of Daegu School of Medicine, Daegu, ${ }^{3}$ Department of Thoracic and Cardiovascular Surgery, St. Vincent's Hospital, College of Medicine, The Catholic University of Korea, Suwon, ${ }^{4}$ Center for Lung Cancer, National Cancer Center, Goyang, ${ }^{5}$ Division of Pulmonology, Department of Internal Medicine, Chungnam National University, Daejeon, ${ }^{6}$ Department of Medicine, Samsung Medical Center, Sungkyunkwan University, Seoul, ${ }^{7}$ Division of Pulmonology, Department of Internal Medicine, The Cancer Research Institute, College of Medicine, The Catholic University of Korea, Seoul, ${ }^{8}$ Department of Cardiothoracic Surgery, Pusan National University Hospital, Busan, ${ }^{9}$ Department of Pulmonary Medicine, Chonnam National University Hwasun Hospital, Chonnam National University Medical School, Hwasun, ${ }^{10}$ Cancer Registration and Statistics Branch, National Cancer Center, Goyang, ${ }^{11}$ Department of Oncology, Asan Medical Center, University of Ulsan College of Medicine, Seoul, Korea

Tuberc Respir Dis. 2019 Apr;82(2):118-125. Published online Jun 19, 2018. https://doi.org/10.4046/trd.2017.0128

On page 121 of this article, there was an error in Figure 1. Three graphs in Figure 1 were shown in the same way.

The correct version of Figure 1 is included below.

We sincerely apologize for this mistake and any inconvenience that we have caused. 
A Total patients

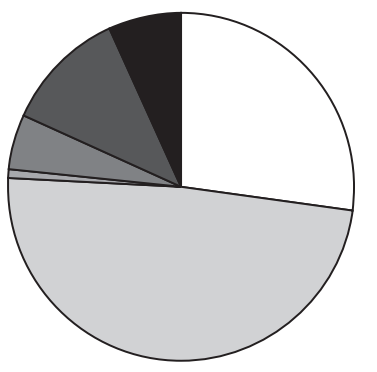

Squamous cell carcinoma $27.2 \%$

Adenocarcinoma $48.7 \%$

Large cell carcinoma $0.6 \%$

NSCLC NOS 5.3\%

Small cell carcinoma $11.5 \%$

Others $6.7 \%$

\section{Female}

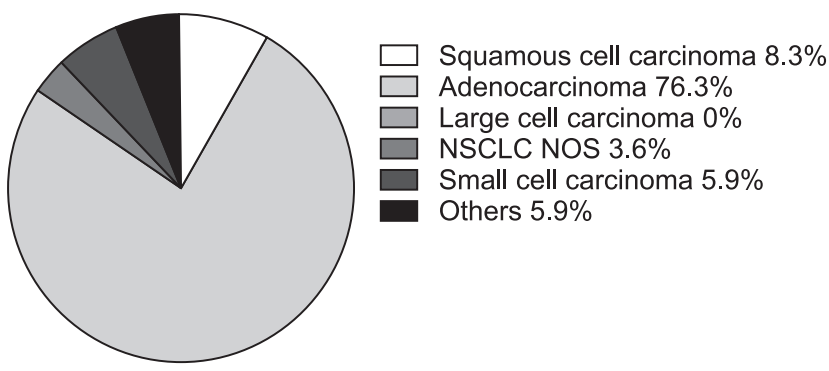

\section{B Male}

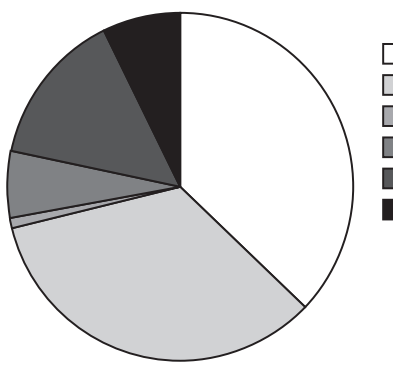

Squamous cell carcinoma $37.2 \%$

Adenocarcinoma $34.1 \%$

Large cell carcinoma $0.9 \%$

NSCLC NOS $6.3 \%$

Small cell carcinoma $14.4 \%$

Others $7.2 \%$

Figure 1. Proportion of lung cancer histological type based on Korean Nationwide Lung Cancer Registry. (A) In total patients. (B) In male sex. (C) In female sex. NSCLC NOS: non-small cell lung cancer not otherwise specified. 